\title{
28 Research Soure \\ Design of Visible Broadband Metamaterial Absorber Based on Metal Nickel
}

\section{Yunji Wang ( $\nabla$ wangyun_ji@126.com )}

Jinling Institute of Technology https://orcid.org/0000-0003-4697-2393

\section{Fei Liu}

Jinling Institute of Technology

Lin Chen

Jinling Institute of Technology

\section{Research Article}

Keywords: metamaterial absorber, Nickel, electrical and magnetic resonance, visible spectra

Posted Date: December 3rd, 2021

DOI: https://doi.org/10.21203/rs.3.rs-1022774/v1

License: (1) This work is licensed under a Creative Commons Attribution 4.0 International License.

Read Full License 


\section{Abstract}

In this paper,we have proposed a visible broadband metamaterial absorber based on metal Nickel. The metamaterial absorber (MA) is composed of three layers,which are Nickel-based pattern array on the top, silicon dioxide in the middle layer and Nickel film at the bottom. In the whole visible spectrum(380$780 \mathrm{~nm}$ ) of our study, the average absorption of the absorber is up to $95.60 \%$. The absorption rates at $500 \mathrm{~nm}$ and $637 \mathrm{~nm}$ are $96.63 \%$ and $99.93 \%$, respectively. Also, the proposed MA is insensitive of polarization in the whole visible regime. Later, the mechanism of the absorption and the influence of the structural parameters on the absorption spectrum are investigated. Strong electrical and magnetic resonance are related to the absorption peak at the resonant wavelength. The results show the resonant wavelength of $500 \mathrm{~nm}$ is fixed and the resonant wavelength of $637 \mathrm{~nm}$ can be tuned by the parameters. The proposed structure will have important application prospects in the field of solar cells.

\section{Introduction}

Since Landy(N. I. Landy et al. 2008)proposed the concept of metamaterial absorber, metamaterial absorbers $\square$ MAs $\square$ have attracted extensive research because of its wide application prospect in such as thermal photovoltaics (Wei Wang et al.2019)

,sensing( Liu, N. et al. 2010, Luo, S. W. et al. 2016), color pringting(F. Cheng et al. 2015, Z. Li et al. 2016, W. Wang et al. 2017), and so on. Metamaterials with different materials and structures can achieve

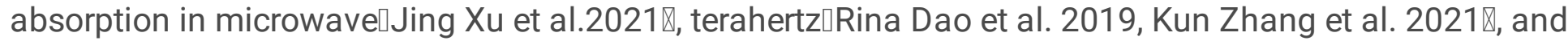
optical wavebandロYang Wang et al. 2021, Xiaoshan Liu et al.2019, Mohammad Mehrabi et al. 2018区. Usually, the metal-dielectric-metal sandwich structure is used to realize the perfect absorption and the absorption spectra is determined by the top metallic pattern layer. Generally, substantial absorbers can be categorized into broadband absorbers (Aydin K. et al.2011, Ding F. et al. 2012, Cui Y. et al. 2012 ) and narrowband absorbers(Li Z. et al. 2014, Meng L. et al. 2014, Lu X. et al.2015 ). Narrowband perfect absorbers can be used in sensing while broadband absorbers are used in thermo-photovoltaics (Zhengdong Yong et al. 2016).

$\mathrm{Ni}$ (Nickel), a kind of non-noble metal, were used to form such metal-dielectric-metal structure to realize a good absorption in the visible spectra(Mehmet Bağmancıa et al. 2017, Minghui Luo et al. 2017). The absorption is because of the nature of this Ni metal. The MAs consisted of $\mathrm{Ni}$ as the metal were designed and fabricated, and achieved polarization-independent absorption of an average level of above $90 \%$ in the whole visible regime(380-780nm)(Minghui Luo et al. 2017).However, the effect of structural parameters on absorption spectra was not investigated. And anther proposed MA showed perfect absorption of $98.4 \%$ at the resonance frequency of $621.76 \mathrm{THz}$ and had also $88.28 \%$ overall absorption in visible frequency regions(Mehmet Bağmancıa et al. 2017). The metallic pattern was complicated and the average absorption needs to increase. 
In this paper, we have designed and proposed a novel broadband perfect metamaterial absorber(MA) in the visible spectra. The influence of the structural parameters on the absorption spectra and the absorption mechanism are carefully investigated. And we have achieve a perfect absorption of $99.89 \%$ at the wavelength of $637 \mathrm{~nm}$ and an average absorption of $95.60 \%$ in the whole visible spectra(380-780nm). The results show that one resonant wavelength is fixed and the other can be tuned by structural parameters.

\section{Brief Theory About The Metamaterials Absorber}

Assume $\mathrm{A}(\omega) \square \mathrm{R}(\omega)$ and $\mathrm{T}(\omega)$ represent the absorption, reflection, and transmission spectra respectively. The absorption $\mathrm{A}(\omega)$ can be induced by $\mathrm{A}(\omega)=1-\mathrm{R}(\omega)-\mathrm{T}(\omega)$ (Mehmet Bağmancıa et al. 2017). However, when the thickness of the bottom Ni metal layer is much larger than its skin depth at the operation frequency, the transmission rate can be ignored $(\mathrm{T}(\omega \approx 0))$. Thus we can obtain $\mathrm{A}(\omega)$ from $A(\omega)=1-R(\omega)$ (Xiyao Chen et al.2014).

And the average absorption rate $\left(A_{a v}\right)$ is calculated by the equation as

$$
A_{a V}=\left(\lambda_{m}-\lambda_{s}\right)^{-1} \int_{\lambda_{s}}^{\lambda_{m}} A(\lambda) d \lambda
$$

1

Where $\lambda$ is the wavelength of the incident wave, and $\lambda_{m}, \lambda_{s}$ are the maximum and minimum wavelengths of the incident wave, respectively(Yang Wang et al.2021).

\section{Results And Discussion}

Figure 2 shows the absorption and reflection spectra of the broadband visible absorber at normal incidence. We can see from the black line in Fig. 2, the proposed MA achieve absorption above $80 \%$ in the whole visible waveband(380-780nm).The two resonant wavelengths are $500 \mathrm{~nm}$ and $637 \mathrm{~nm}$. And the absorption rate at $637 \mathrm{~nm}$ is $99.93 \%$. Also the average absorption rate is up to $95.60 \%$ calculated by equation (1).And Fig. 3 shows the simulated adsorption spectra of proposed MA for cases of TE and TM polarization. It is obvious that the proposed MA is insensitive to the polarization.

In order to well-recognized the mechanism of the resonant absorption, the distributions of the electric field of $E_{z}$ at the surface of metal arrays at the two wavelength,500nm and $637 \mathrm{~nm}$ for TE and TM , are depicted in Fig. 4.We can see that the strong electric field is distributed at the four cylinders and the rectangular strip perpendicular to the polarization direction both at $500 \mathrm{~nm}$ and $637 \mathrm{~nm}$.Fig. 5 shows the magnetic field of $\mathrm{H}_{\mathrm{z}}$ at the top surface.It is found that strong magnetic field is distributed at the four cylinders and the rectangular strip perpendicular to the polarization direction at $500 \mathrm{~nm}$, however, strong magnetic field 
is distributed at all the graphic arrays at $637 \mathrm{~nm}$ for both TE and TM incidence. All these imply strong electric and magnetic resonance absorption at the graphic arrays on top.

Evidently, the absorption spectra strongly depend on the geometric parameters of the resonators. To confirm the flexibility of design, we discuss the effects of each geometric parameter on the absorption behavior of the proposed MA unit cell.In order to have a better understanding, the absorption curves achieved by changing the different parameters,such as dielectric layer thickness, resonator thickness, cross length and cylinder radius, are given in Fig. 6-Fig. 7.

First, the dielectric thickness $\left(h_{2}\right)$ and the resonator thickness $\left(h_{1}\right)$ has been changed around the reference value.As we can see from Fig. 6(a),the dielectric thickness varies from $40 \mathrm{~nm}$ to $70 \mathrm{~nm}$ with a step of $10 \mathrm{~nm}$. There are three absorption peaks in the band from $100 \mathrm{~nm}$ to $780 \mathrm{~nm}$. There is a narrow peak between 141 and $181 \mathrm{~nm}$,and it shifts slightly red with the increase of silica layer thickness. The absorption peak at $500 \mathrm{~nm}$ is almost unchanged with the change of silica layer thickness,while the other absorption peak shifts red from $382 \mathrm{~nm}$ to $689 \mathrm{~nm}$ with the increase of silica layer thickness from 40nm to $70 \mathrm{~nm}$. The waveband we are interested in is $430 \mathrm{~nm}$ to $770 \mathrm{~nm}$ which covers the whole visible light.It can be seen from Fig. $6(\mathrm{~b})$ that there is an absorption peak in the whole visible band before the pattern thickness is $15 \mathrm{~nm}$. With the increase of the pattern thickness,the position of the absorption peak remains unchanged and the absorption rate increase slightly. There are two absorption peaks after the pattern thickness is greater than $15 \mathrm{~nm}$. With the increase of the pattern thickness, the absorption peak of $500 \mathrm{~nm}$ still remains unchanged but the absorption rate decrease from $99.9 \%$ of $15 \mathrm{~nm}$ thickness to $91.2 \%$ of 30 $\mathrm{nm}$ thickness.Another absorption peak appears after the pattern thickness is greater than $15 \mathrm{~nm}$ and the absorption rate remains above $99.9 \%$. However,the position of the absorption peak shifts red from $637 \mathrm{~nm}$ to $681 \mathrm{~nm}$ with the increase of the pattern thickness from $20 \mathrm{~nm}$ to $30 \mathrm{~nm}$.

Second, the absorption curves of proposed structure according to the different cylinder radius and the rectangular strip lengths are depicted in Fig. 7(a) and (b).We can see from Fig. 7.(a),before the cylinder radius is $40 \mathrm{~nm}$, there is one absorption peak which stays unchanged with the change of the cylinder radius. However,the absorptivity in the whole visible band increases with the increase of the cylinder radius.Double absorption peaks appear when the cylinder radius is greater than $40 \mathrm{~nm}$. The absorption rate at $500 \mathrm{~nm}$ decreases with the increase of the cylinder radius, the other absorption peak shifts red from $567 \mathrm{~nm}$ to $713 \mathrm{~nm}$, and the absorption rate decrease slightly while the bandwidth with an absorptivity greater than $90 \%$ increase greatly.It can be seen that changing the radius of the cylinder can adjust the position and bandwidth of one of the absorption peaks. From Fig. 7.(b), we find that with the increase of the rectangular strip of the cross, the position of the absorption peak at $500 \mathrm{~nm}$ remains unchanged and the peak absorption rate decreases. The absorption peak at $637 \mathrm{~nm}$ shifts red and decreases sightly.

In the end, we simulated the absorption curves of only cross-shaped resonator,only-cylinders resonator ,no-pattern and proposed structure in Fig. 8. Compared with no-pattern structure,all the MAs have a better performance in visible band and there is a absorption peak at $500 \mathrm{~nm}$ which is because of the nature of 
the metal Ni.Another absorption peak at $637 \mathrm{~nm}$ is because of the coupling effect of the cross and four cylinders.We can have the inclusion that our proposed MA have a perfect absorptivity.

\section{Conclusions}

In this paper, we have proposed a MA which have a perfect absorption in the visible band. The MA consists of three layers, metallic pattern on top, silica in the middle and the metal film at the bottom. The electric and magnetic field distributions show that the strong absorption is because of the electric and magnetic resonance.We can see two absorption peak in the whole visible band.The position of the $500 \mathrm{~nm}$ peak remains unchanged with the changing of the structural parameters, while the other can be tuned with the changing of the structural parameters. The MA we proposed can apply to the field of solar cells.

\section{Declarations}

\section{Acknowledgments}

This research was funded by the Ph.D. Project supported by the Jinling Institute of Technology (Grant No. jit-b-201902).

\section{Competing financial interests}

The authors declare no competing financial interests.

\section{References}

1. N. I. Landy, S. Sajuyigbe, J. J. Mock, D. R. Smith, W. J. Padilla, S. Sajuyigbe, and D. J. Mock, Perfect metamaterial absorber, Phys. Rev. Lett. 100(20), 207402 (2008). DOI: 10.1103/PhysRevLett.100.207402

2. Wei Wang , Xiaodong Yang, et al.Enhanced quantum dots spontaneous emission with metamaterial perfect absorbers[J]Applied Physics Letters, 2019,114:021103. DOI: 10.1063/1.5081688

3. Liu, N., Mesch, M., Weiss, T., Hentschel, M. \& Giessen, H. Infrared perfect absorber and its application as plasmonic sensor. Nano Lett,2010,10:2342-4348 DOI: 10.1021/nl9041033

4. Luo, S. W., Zhao, J., Zuo, D. L. \& Wang, X. B. Perfect narrow band absorber for sensing applications. Opt. Express,2016,24:9288-9294 DOI:10.1364/OE.24.009288

5. Fei Cheng, Xiaodong Yang, Daniel Rosenmann, Liliana Stan, David Czaplewski, and Jie Gao. Enhanced structural color generation in aluminum metamaterials coated with a thin polymer layer. Opt. Express,2015, 23: 25329-25339 DOI:10.1364/OE.23.025329

6. Zhigang Li, Wei Wang, Daniel Rosenmann, David A.Czaplewski, Xiaodong Yang, and Jie Gao. Allmetal structural color printing based on aluminum plasmonic metasurfaces. Opt. Express,2016, 24: 20472-20480. DOI:10.1364/OE.24.20472 
7. Wei Wang, Daniel Rosenmann, David A.Czaplewski, Xiaodong Yang, and Jie Gao. Realizing structural color generation with aluminum plasmonic V-groove metasurfaces. Opt. Express,2017, 25: 2045420465. DOI:10.1364/OE.25.020453

8. Jing Xu囚Yuancheng Fan, Xiapeng Su,Jing Guo,Jiaxing Zhu,Quanhong Fu,and Fuli Zhang, Broadband and wide angle microwave absorption with optically transparent metamaterial, Optical Materials 113(2021)110852. DOIه10.1016/j.optmat.2021.110852

9. Rina Dao,Xinru Kong,Haifeng Zhang,and Xinran Su,A tunable broadband terahertz metamaterial absorber based on the vanadium dioxide,Optik-International Journal for Light and Electron Optics 180(2019)619-625. DOI:10.1016/j.jijleo.2018.12.004

10. Kun Zhang,Tianyou Ma et al.Dynamically tunable and polarization-insensitive dual-band terahertz metamaterial absorber based on TiNi shape memory alloy films.Results in Physics 23(2021)104001. DOI:10.1016/j.rinp.2021.104001

11. Yang Wang, Xue-Fei Xuan,et al.Numerical study of an ultra-broadband, wide-angle, polarizationinsensitive absorber in visible and infrared region.Optical Materials 114 (2021) 110902.

DOI:10.1016/j.optmat.2021.110902

12. Xiaoshan Liu,Guolan Fu et al. Titanium nanoholes meta-surface for ultra-broadband infrared adsorption.Results in Physics 15(2019)102578. DOI:10.1016/j.rinp.2019.102578

13. Mohammad Mehrabi, Hamid Rajabalipanah et al. Polarization-insensitive,ultra-broadband,and compact metamaterial-inspired optical absorber via wide-angle and highly efficient performances.Applied Optics vol.57,No.14(2018) DOI: 10.1364/A0.57.003693

14. Aydin, K., Ferry, V. E., Briggs, R. M. \& Atwater, H. A. Broadband polarization-independent resonant light absorption using ultrathin plasmonic super absorbers. Nature Commun. 2, 517 (2011). DOI:10.1038/ncomms1528

15. Ding, F., Cui, Y., Ge, X., Jin, Y. \& He, S. Ultra-broadband microwave metamaterial absorber. Appl. Phys. Lett. 100,103506 (2012). DOI:10.1063/1.3692178

16. Cui, Y. et al. Ultrabroadband light absorption by a sawtooth anisotropic metamaterial slab. Nano Lett. 12,1443-1447 (2012). DOI:10.1021/nl204118h

17. Li, Z., Butun, S. \& Aydin, K. Ultranarrow band absorbers based on surface lattice resonances in nanostructured metal surfaces. ACS Nano8, 8242-8248 (2014). DOI:10.1021/nn502617t

18. Meng, L. et al. Optimized grating as an ultra-narrow band absorber or plasmonic sensor. Opt. Lett. 39, 1137-1140 (2014). DOI:10.1364/OL.39.001137

19. Lu, X., Zhang, L. \& Zhang, T. Nanoslit-microcavity-based narrow band absorber for sensing applications. Opt. Express23,20715-20720 (2015). DOI:10.1364/OE.23.020715

20. Zhengdong Yong, Senlin zhang $₫$ Chensheng Gong and Sailing He. Narrow band perfect absorber for maximum localized magnetic and electric field enhancement and sensing application. Scientific Reports 2016, 6:24063 DOI:10.1038/srep24063

21. Mehmet Bağmancıa, Muharrem Karaaslan et al.Broad-band polarization-independent metamaterial absorber for solar energy harvesting applications.Physica E 90(2017)1-6. DOIX 
10.1016/j.physe.2017.03.001

22. Minghui Luo, Su Shen et al. Broadband,wide angle,and polarization-independent metamaterial absorber for the visible regime.Optics Express Vol.25,No.14(2017)16715. DOIV 10.1364/OE.25.016715

23. Xiyao Chen,Yuangang Zhong et al. A tunable infrared plasmonic polarization filter with asymmetrical cross resonator.Chin.Phys.B Vol.23,No.8(2014) 087806 DOI:10.1088/1674-1056/23/8/087806

\section{Figures}

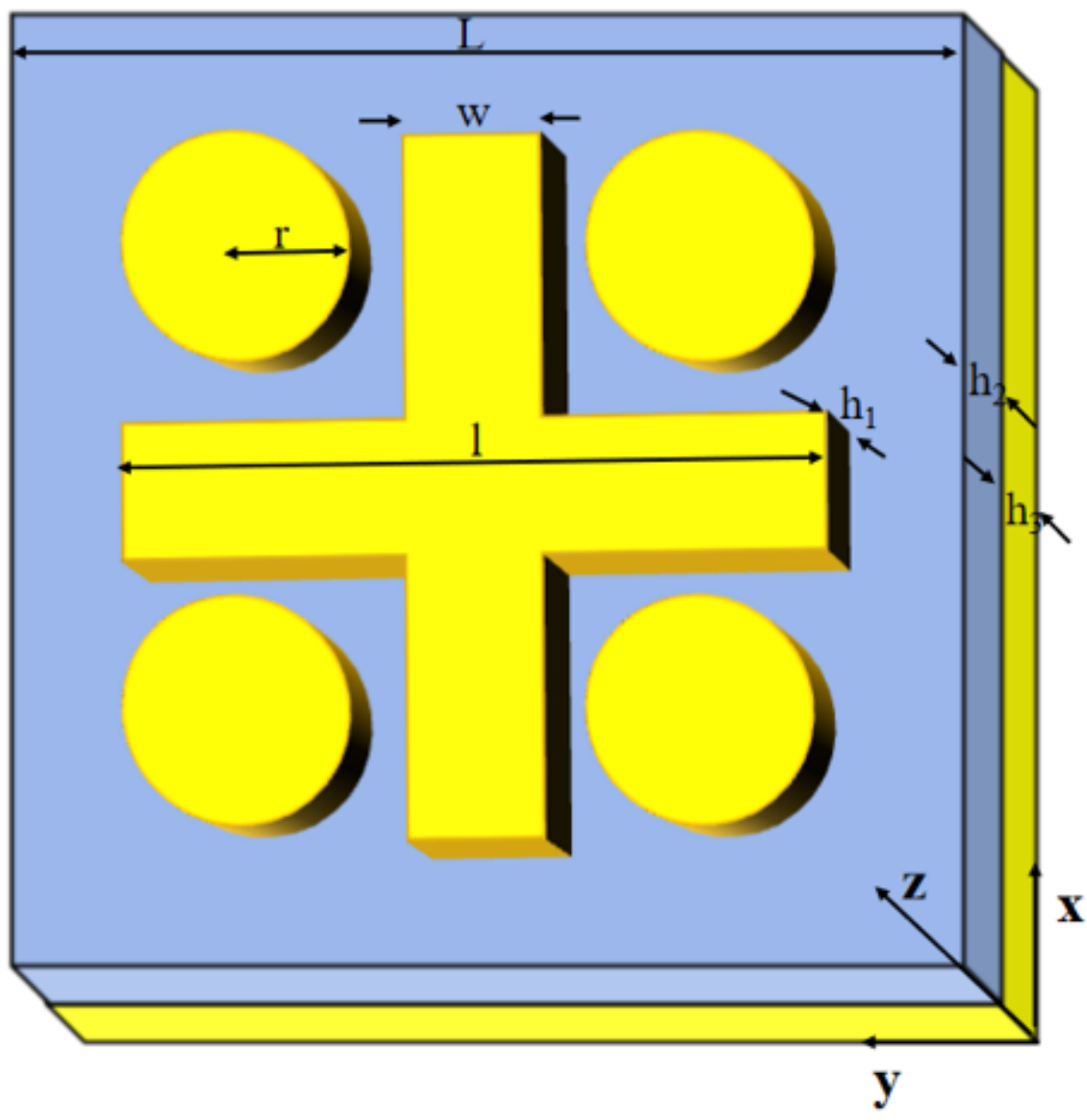

Figure 1

Unit cell of the proposed structure

\section{Figure 2}

The absorption and reflection spectra of the proposed MA in visible 


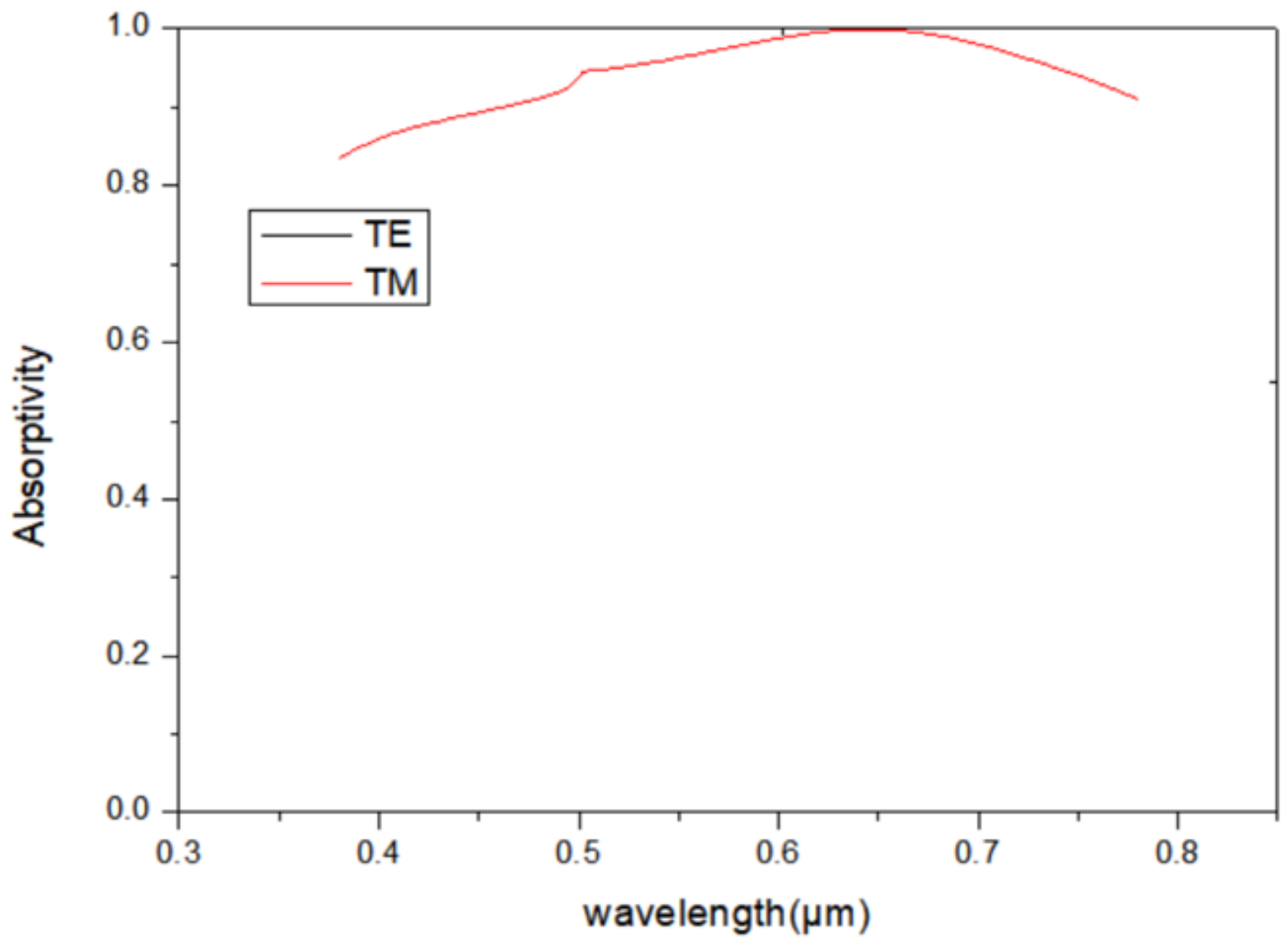

Figure 3

Simulated absorption spectra of proposed MA for cases of TE and TM polarization 

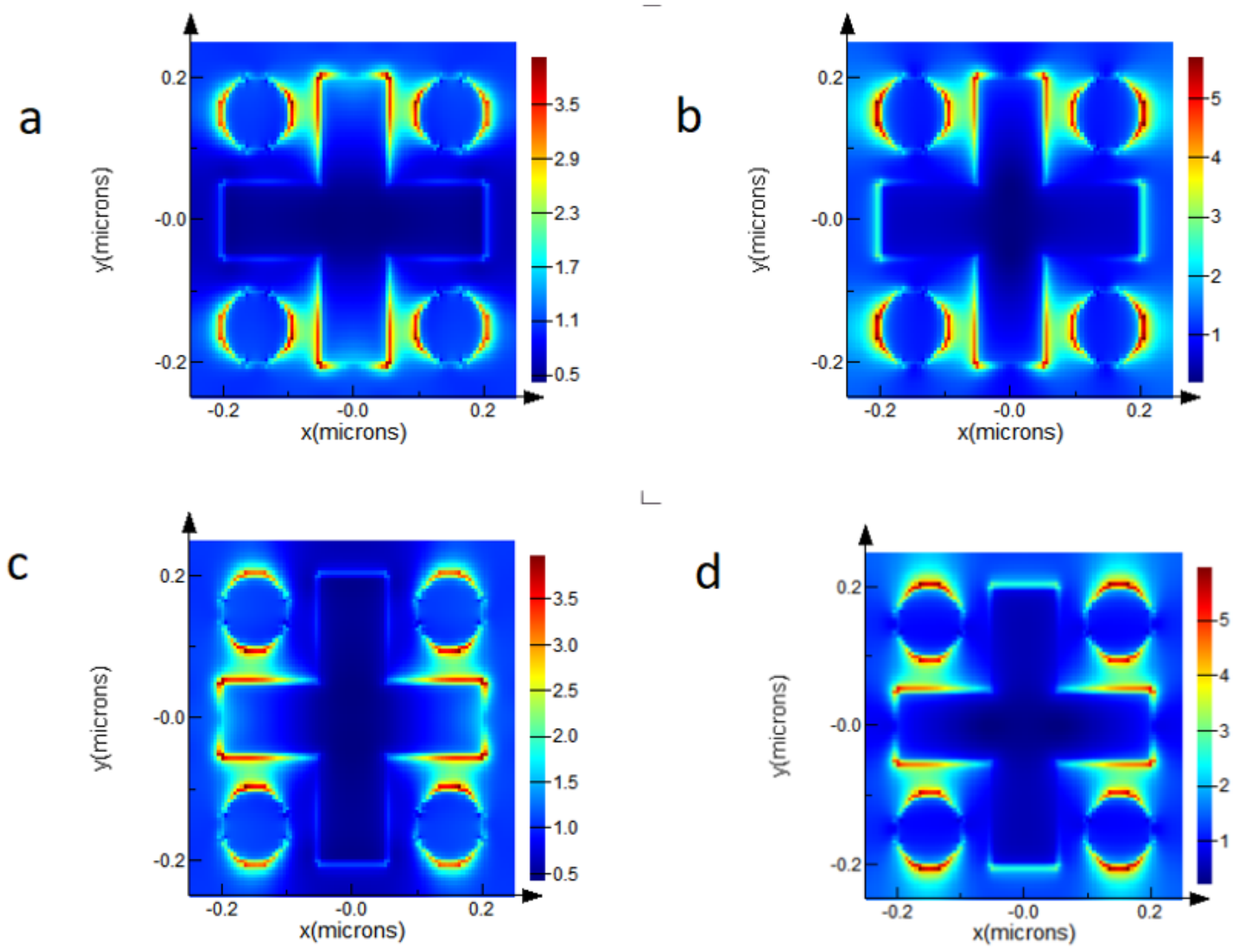

Figure 4

Distributions of the electric field Ez at the surface of the metal array corresponding to TE polarization at the resonant wavelngth a $500 \mathrm{~nm}$ and $\mathrm{b} 637 \mathrm{~nm}$ and corresponding to TM polarization at the resonant wavelength c 500nm and d 637nm 

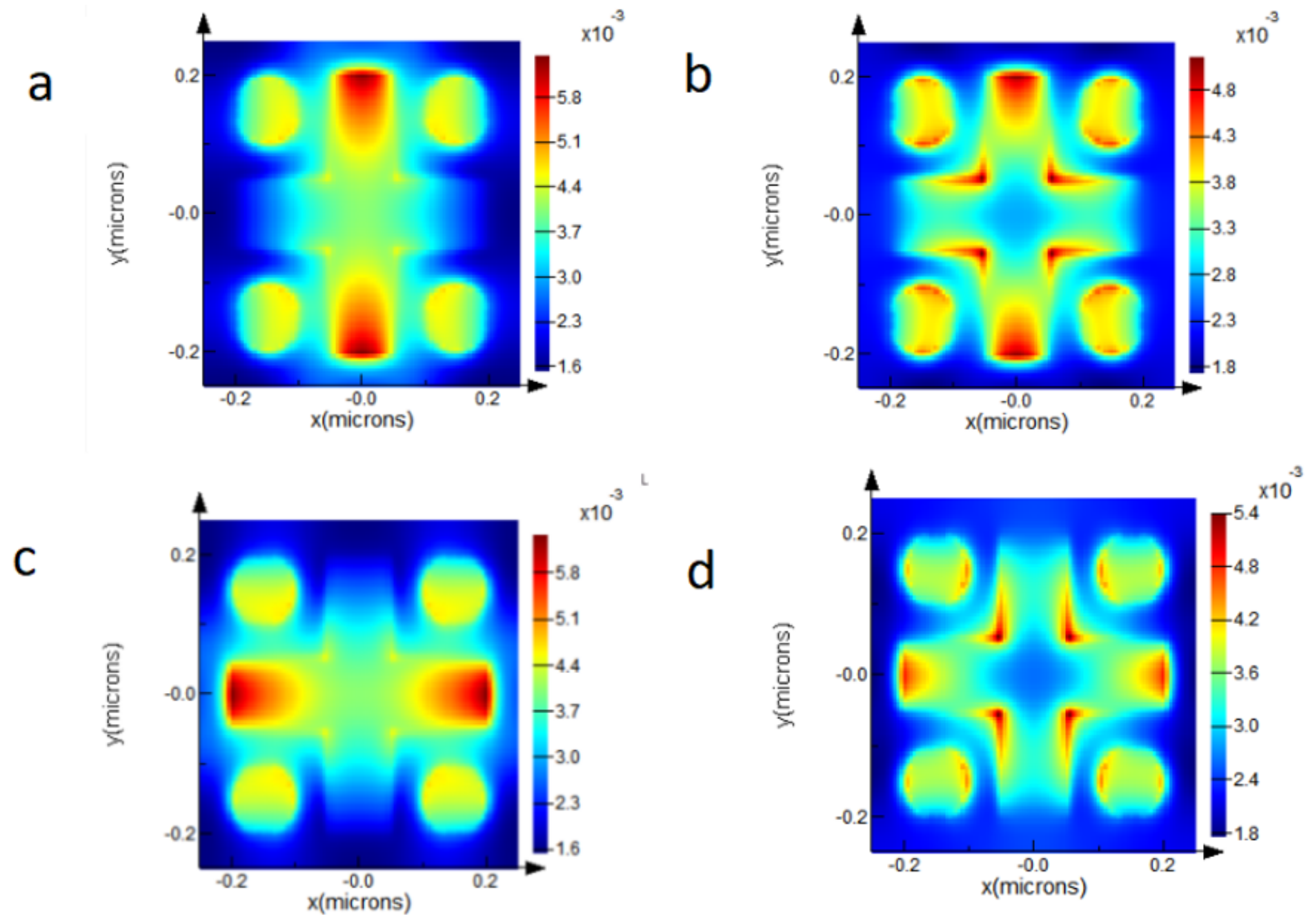

Figure 5

Distributions of the magnetic field $\mathrm{Hz}$ at the surface of the metal array corresponding to TE polarization at the resonant wavelngth a $500 \mathrm{~nm}$ and $\mathrm{b} 637 \mathrm{~nm}$ and corresponding toTM polarization at the resonant wavelength c 500nm and d 637nm. 


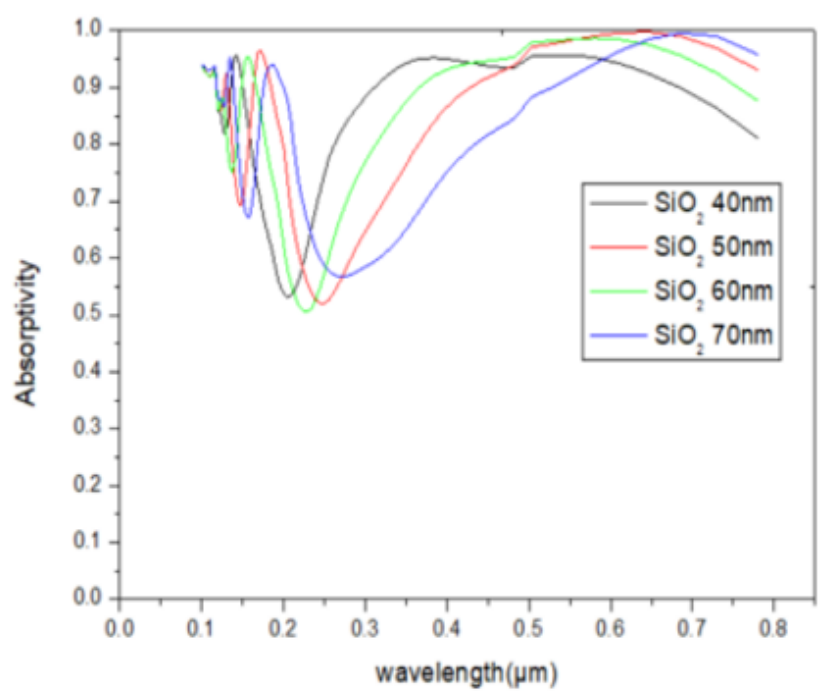

a

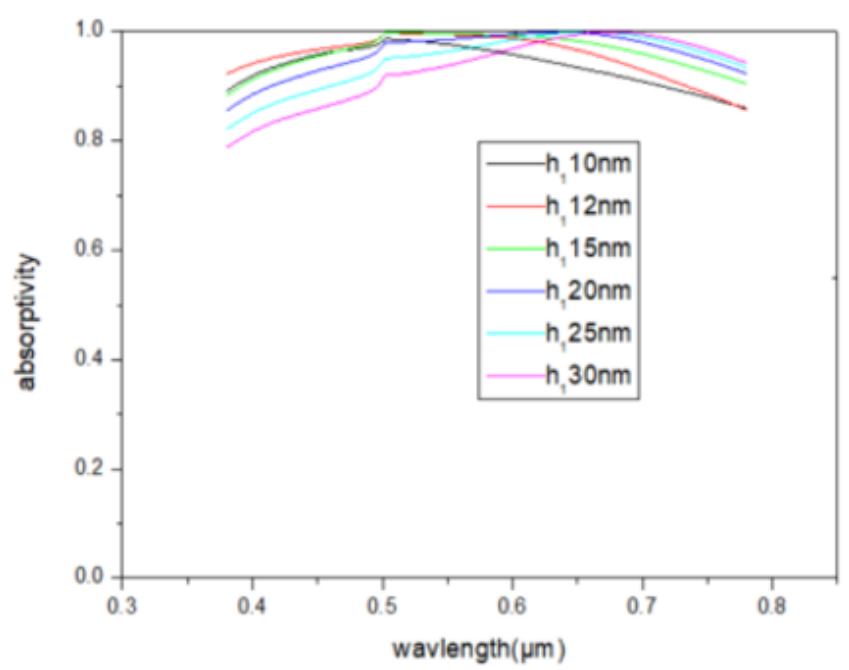

b

\section{Figure 6}

The absorption spectra of proposed structure according to the different (a)dielectric thichness(from $40 \mathrm{~nm}$ to $70 \mathrm{~nm}$ ) (b) resonant thickness(from $10 \mathrm{~nm}$ to $30 \mathrm{~nm}$ )

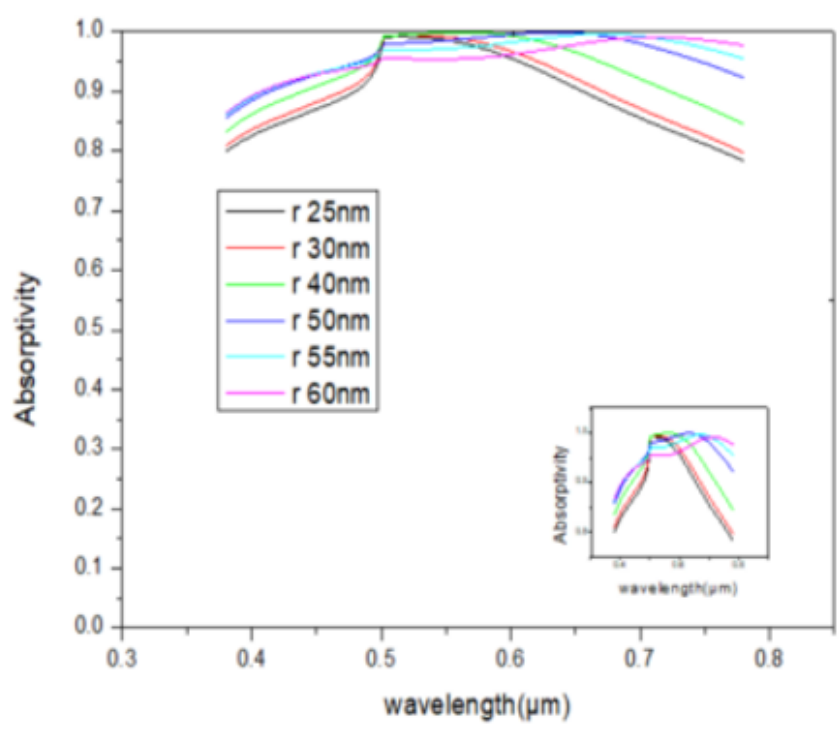

a

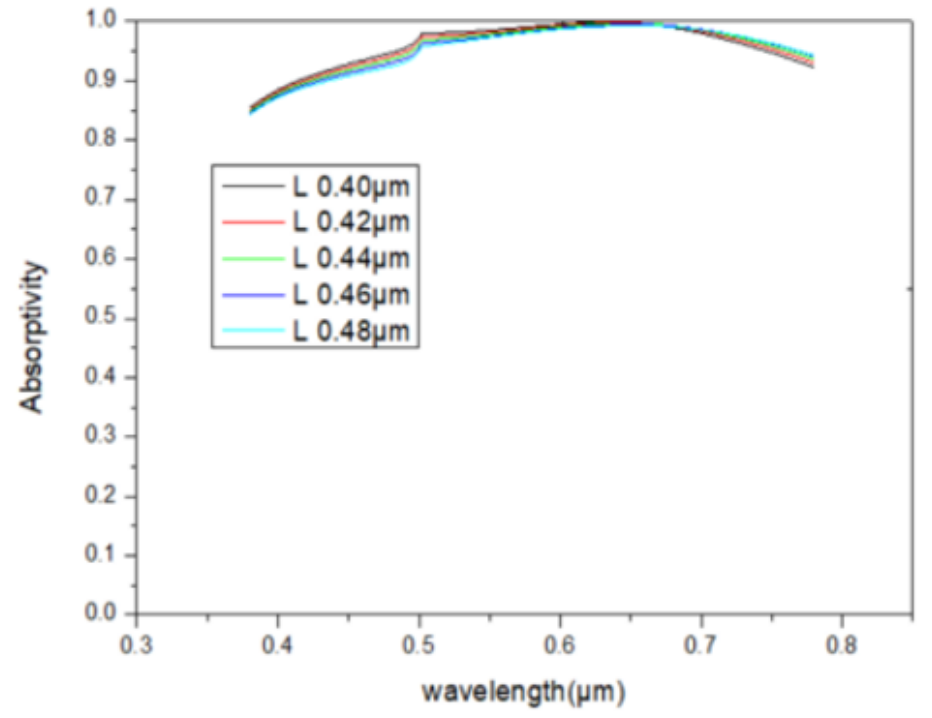

b

\section{Figure 7}

The absorption spectra of proposed structure according to the different (a)cylinder radius(from $25 \mathrm{~nm}$ to $60 \mathrm{~nm}$ ) (b)the rectangular strip length(from $400 \mathrm{~nm}$ to $480 \mathrm{~nm}$ ) 


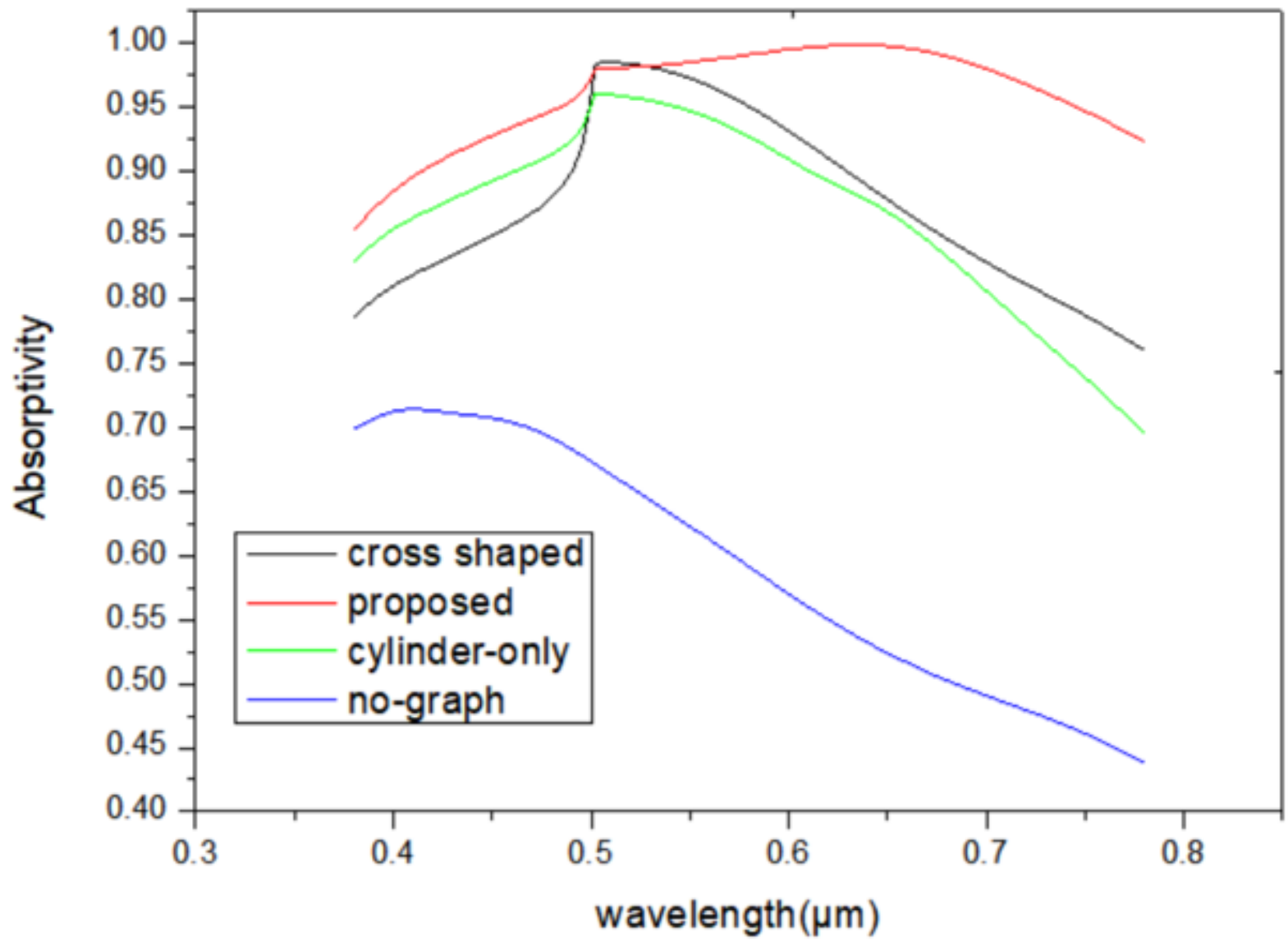

Figure 8

Absorption characteristics for cases of only cross shaped resonator,only cylinders, no-graph and proposed structure. 